\title{
Corela
}

Cognition, représentation, langage

6-1 | 2008

Vol. $6, n^{\circ} 1$

\section{Vers la construction de descriptions argumentées d'un accident de la route : analyse de diverses stratégies argumentatives}

Sara Boutouhami et Daniel Kayser

\section{(2) OpenEdition}

\section{Journals}

Édition électronique

URL : http://journals.openedition.org/corela/234

DOI : $10.4000 /$ corela.234

ISSN : 1638-573X

Éditeur

Cercle linguistique du Centre et de l'Ouest - CerLICO

\section{Référence électronique}

Sara Boutouhami et Daniel Kayser, « Vers la construction de descriptions argumentées d'un accident de la route : analyse de diverses stratégies argumentatives », Corela [En ligne], 6-1 | 2008, mis en ligne le 24 juin 2008, consulté le 19 avril 2019. URL: http://journals.openedition.org/corela/234 ; DOI 10.4000/corela.234

Ce document a été généré automatiquement le 19 avril 2019.

\section{(c) (i) (3)}

Corela - cognition, représentation, langage est mis à disposition selon les termes de la licence Creative Commons Attribution - Pas d'Utilisation Commerciale - Partage dans les Mêmes Conditions 4.0 International. 


\title{
Vers la construction de descriptions argumentées d'un accident de la route : analyse de diverses stratégies argumentatives
}

\author{
Sara Boutouhami et Daniel Kayser
}

\section{Introduction}

1 L'intérêt pour l'argumentation, ou « rhétorique des conflits » (Lempereur, 1991), n'est pas neuf. Cette discipline est étroitement liée à l'histoire de la philosophie, de la rhétorique et du discours. L'argumentation désigne souvent l'échange discursif effectif par lequel des interlocuteurs tentent de défendre une position ou de faire accepter un point de vue. Elle est liée à l'échange de paroles, au dialogue. Plus largement, l'argumentation est un champ d'études à la fois descriptif et critique qui s'intéresse à la mise en forme des arguments (oralement ou par écrit) en vue, notamment, de la persuasion d'un auditoire.

2 Il n'existe pas de définition universellement acceptée de l'argumentation. Cette notion floue, qui parait simple, s'avère assez complexe dès qu'on essaye de la définir. L'argumentation peut évoquer plusieurs notions que nous énumérons en vrac: la communication, la persuasion, la logique, le raisonnement, le discours, le dialogue, la langue naturelle, les normes sociales, la rationalité, la vérité, la vraisemblance, la démonstration, la preuve, le conflit, le désaccord, ... Son rôle central dans les différentes formes de communications humaines explique l'intérêt qu'elle a suscité chez les chercheurs en Intelligence Artificielle (IA). Les travaux qui ont été effectués dans ce domaine se sont principalement donné pour but de proposer des cadres formels qui rendent compte entre autres de notions comme celles d'argument, de contre-argument ou de force d'un argument dans une situation donnée (Dung, 1995). Ces travaux utilisent le plus souvent les outils logiques et se basent ainsi largement sur la notion de vérité. Malgré leur intérêt théorique et pratique, ces travaux ne reflètent pas fidèlement la 
nature spontanée de l'argumentation telle qu'elle est employée dans les formes habituelles de communication humaine, notamment la forme orale et la forme écrite qui nous intéresse ici.

3 D'une façon générale, les travaux sur l'argumentation sont nombreux, et tenter de les classifier est une tâche très délicate. Comme le souligne (Baker, 1994), Plantin (1990) distingue des problématiques de l'argumentation selon qu'il s'agit d'un fait de la langue (Ducrot, 1980 ; Anscombre \& Ducrot, 1983) ou bien du discours ; et dans cette dernière catégorie, selon que son but est d'établir une vérité, par un raisonnement logique ou scientifique, formel (Grize, 1990 ; Amgoud \& al., 2005) ou informel (Toulmin, 1958), ou qu'on agit dans un but d'efficacité (rhétorique) (Perelman \& Olbrechts-Tyteca, 1985).

4 Le champ d'application de l'argumentation s'étale sur de nombreux domaines : parmi ceux qui ont fait l'objet de travaux récents, on citera le domaine juridique (Prakken \& Sartor, 1996 ; Prakken, 1997), le domaine médical (Das \& al., 1996), la négociation entre agents (Sycara, 1989) et la prise de décision (Garcia \& al., 2000).

Dans notre travail, nous nous intéressons à l'expression d'argumentations en Langue Naturelle (LN). Nous ne dissocions pas les arguments de leur cadre d'expression (le texte) mais nous considérons, au contraire, que les particularités de ce cadre sont au cœur du processus d'argumentation. Notre étude de l'argumentation est étroitement liée à la nature du processus de compréhension de la langue naturelle. En effet, nous nous plaçons dans une vision inférentielle de la langue dans laquelle la compréhension d'un texte est assimilée aux conclusions qu'un lecteur humain peut tirer de sa lecture par un raisonnement de bon sens basé sur sa connaissance du domaine. Dans cette optique, le but de l'argumentation d'un auteur est de déclencher des inférences du lecteur conduisant finalement ce dernier à conclure ce qui «arrange» l'auteur. L'enjeu sera donc d'étudier par quels moyens et quelle forme stratégique on peut générer des descriptions textuelles argumentées d'une même réalité effective, sans pour autant travestir cette réalité. Il convient de noter que ces moyens et ces stratégies touchent à tous les niveaux de la langue: choix lexical, tournure syntaxique, style, ordre des phrases, rien n'est neutre et il faut faire le meilleur usage possible de tous les artifices que permet la langue pour produire l'effet voulu.

6 En fait, aucune description textuelle ne fournit une image exhaustive de la réalité : ceci donnerait des textes excessivement longs et fastidieux ; l'auteur compte sur les capacités inférentielles du lecteur pour combler certains détails, ce qui laisse une marge de manœuvre importante. Notre but est d'utiliser cette marge de manœuvre en introduisant un biais en faveur de l'auteur. En d'autres termes, nous voulons orienter le raisonnement du lecteur de manière à ce qu'il comble la description de la façon qui soit la plus favorable possible à l'auteur : c'est ce que nous appelons une description argumentée. La production de nombreuses descriptions argumentées d'un même événement est possible parce qu'on peut choisir de différentes manières les "trous " que le raisonnement nécessaire à la compréhension de la LN viendra combler, en recourant à un vaste ensemble de normes du domaine pour inférer des conclusions plausibles à partir du texte.

7 Dans la section 2, nous décrivons avec plus de précision notre tâche et nous présentons le domaine d'application choisi : celui des accidents de la route. La section 3 est consacrée à la présentation de la méthodologie que nous proposons pour la génération d'une description argumentée, en discutant les différentes parties de l'architecture d'un système que nous développons actuellement à cette fin. Dans la section 4 , nous donnons quelques éléments du langage formel que nous utilisons pour la représentation des 
connaissances et le raisonnement dans notre système. Enfin, une conclusion de ce travail ainsi que quelques pistes d'investigations futures sont présentées dans la section 5.

\section{Motivation du choix et description de la tâche}

Pour construire une description argumentée, il faut un point de départ. Nous supposons donc disposer d'une description "complète et neutre» d'un événement qui s'est réellement produit. Notre objectif est de nous servir de cette description pour construire un texte qui :

- i. contient suffisamment d'information pour que son lecteur puisse reconstituer les grandes lignes de cet événement,

- ii. ne contient aucune contre-vérité,

- iii. donne au lecteur la meilleure image possible du comportement d'un des protagonistes de l'événement.

9 Le point (ii) mérite immédiatement un commentaire : il implique évidemment que la description argumentée ne doit contenir aucun fait matériel contradictoire avec ce qui est rapporté dans la description " complète et neutre ». Mais l'événement ne s'explique que par une succession, dans la tête des protagonistes, d'intentions et de croyances confirmées ou démenties par les faits ultérieurs; et là, nous nous autorisons une certaine latitude : la description argumentée pourra supposer que l'un ou l'autre des acteurs de l'événement avait des intentions ou des croyances qui ne sont fondées sur aucun élément de la description neutre, et ne sont donc peut-être pas conformes à la vérité.

Nous avons choisi comme champ d'application le domaine des accidents de la route, car notre équipe a déjà une expertise dans ce domaine (Tal, 1994). Comme on sait, les conducteurs impliqués dans un accident sont amenés à rédiger, au verso des «constats amiables», des rapports textuels courts décrivant les circonstances de l'accident, qui sont destinés à leur compagnie d'assurances. Quoique celles-ci semblent n'utiliser qu'exceptionnellement ces textes, leurs auteurs pensent qu'ils servent à déterminer leurs responsabilités. Ils essaient donc naturellement de présenter les choses de la manière la plus avantageuse pour eux, et on observe dans le corpus que nous avons recueilli ${ }^{1}$ le recours à diverses techniques d'argumentation. Le choix de ce domaine se justifie aussi par le fait qu'il fait appel à un contexte lexical et sémantique assez restreint et à un ensemble de normes dont nous avons identifié une grande partie. De plus, les situations d'accident sont variées ce qui permet d'alimenter largement notre étude par des cas et des besoins d'argumentation divers.

11 Dans un premier temps, nous avons analysé les stratégies argumentatives utilisées par les auteurs de ces rapports, et le présent article, tout en présentant les grandes lignes des phases suivantes, rend principalement compte de cette analyse préalable. En effet, l'impression plus ou moins favorable que nous ressentons à la lecture de ces rapports nous permet de formuler des hypothèses sur la valeur argumentative de certains procédés, et nous serons à même de tester ces hypothèses en les utilisant dans notre travail de génération.

Une première constatation est que les auteurs ne sont pas toujours très convaincants, et notre ambition est de générer des textes qui seront jugés, par des lecteurs non prévenus, 
mieux défendre la cause de leur auteur. En effet, certains rédacteurs, sûrs d'être dans leur droit, insistent à tel point sur la qualité de leur comportement et sur les erreurs de leur adversaire qu'ils en deviendraient presque suspects. D'autres au contraire, se sachant dans leur tort, se contentent d'exploiter les ressources lexicales de la langue pour choisir des mots et des expressions qui minimisent leur responsabilité.

Pour pouvoir comparer ce que nous produirons avec les textes de notre corpus, il faut être assuré de partir d'une même réalité. Or nous n'avons évidemment pas accès aux circonstances réelles de l'accident (nous ne connaissons celui-ci que par ce que les auteurs veulent bien nous en dire). Il nous est donc impossible de partir d'une "description complète et neutre " de ce qui s'est passé. La solution adoptée est de fabriquer à partir des textes du corpus une description de ce qu'à tort ou à raison (peu importe en définitive), nous estimons avoir été le déroulement réel de l'accident.

Notre contribution consiste à articuler, dans une même architecture, les différents moyens inférentiels et lexicaux qu'on peut mettre au service de l'argumentation dans une situation donnée. Le corpus nous sert ainsi à la fois comme source d'inspiration de diverses situations d'accidents et comme référence pour évaluer les résultats de notre système : pour un accident donné, le but sera de produire des descriptions aussi bien ou « mieux » argumentées que celles contenues dans ce corpus.

\section{Une méthodologie pour l'argumentation}

Notre point de départ est donc une description détaillée d'un accident de la route. Cette description contient l'ensemble de tous les faits relatifs à l'accident: nous avons inféré ces faits en nous basant sur un rapport authentique de notre corpus ; comme nous l'avons dit, il est tout à fait possible, et même probable, que ces inférences ne correspondent pas exactement à la façon dont les choses se sont déroulées, mais ceci n'a pas de véritable incidence sur notre travail. Bien, mal, ou pas argumentée du tout, une description doit avant tout rapporter les faits jugés indispensables pour comprendre comment l'accident a eu lieu. Ils appartiennent alors à une liste nommée liste minimale. Un fait appartient à la liste minimale si et seulement s'il n'est pas inférable à partir du reste des faits rapportés et qu'en son absence, nous ne pouvons plus comprendre le déroulement de l'accident. Pour le moment, la détermination de cette liste se fait manuellement.

Nos données consistent donc en une liste complète de propositions, au sein de laquelle nous distinguons une liste minimale de faits dont nous considérons que, sous une forme ou sous une autre, ils sont indispensables à toute description de l'accident. Notre objectif est de construire une liste ordonnée de prédications qui serviront de trame à la rédaction $\mathrm{du}$ « meilleur rapport possible » pour le point de vue d'un des protagonistes de l'accident. Nous n'irons probablement pas jusqu'à la rédaction effective de ce rapport, pour ne pas avoir à aborder les problèmes connus et relativement bien résolus que l'on rencontre en génération de textes, notamment les questions d'accord, de concordance, de reprise anaphorique, car ces questions ne sont pas spécifiques à notre étude.

\subsection{Les statuts argumentatifs}

Dans une première étape, nous attribuons un "statut argumentatif» à chaque proposition de la liste. Le statut argumentatif d'un fait donné permet de déterminer la façon dont il 
faut le présenter pour qu'il contribue positivement à notre but d'argumentation. On distingue trois principaux statuts argumentatifs :

\section{Bon à dire} faveur de l'auteur; ils vont figurer explicitement dans la description argumentée, sauf si leur abondance ou leur redondance risque d'entraîner une réaction de saturation du lecteur. Un fait donné peut recevoir ce statut dans deux cas de figure :

- il s'agit d'une «bonne» action effectuée par l'auteur, dans le sens où elle reflète le respect d'une certaine norme.

- ce peut aussi être une «mauvaise» action effectuée par son adversaire i.e. la violation d'une norme (exprimer des violations de normes par l'adversaire est un procédé argumentatif efficace, qui souligne par contraste le bon comportement de l'auteur).

\section{Taire (passer sous silence)} que le fait de le mentionner n'est pas indispensable à la compréhension du texte (nous ne l'avons pas inclus dans notre «liste minimale»), alors il vaut mieux ne rien dire à son propos. En fait, en examinant quelques textes de notre corpus, nous avons constaté que plusieurs d'entre eux sont un peu faibles du point de vue argumentatif car leur auteur met trop d'informations inutiles qui nuisent à son argumentation. Le silence fait partie des stratégies argumentatives, et c'est parfois la meilleure d'entre elles.

\section{Sous-entendre (insinuer)}

Si un fait n'est pas en faveur de l'auteur, ou bien qu'il est en faveur de son adversaire et qu'il appartient à la liste minimale, ce fait doit donc figurer dans la description argumentée. Mais il peut n'y figurer qu'implicitement; en d'autres termes, on peut l'«insinuer» afin que le lecteur l'infère sans que l'auteur n'ait à le dire ouvertement. Il n'est cependant pas toujours possible de pouvoir insinuer un fait au lieu de l'expliciter. Dans de tels cas, l'ultime solution est de faire appel à des techniques lexico-syntaxiques pour formuler ce fait d'une façon qui minimise autant que possible son impact sur le lecteur.

\subsection{Architecture générale du système d'argumentation}

La figure 1 représente l'architecture générale de notre système. À partir des données (liste complète et liste minimale), des règles générales permettent de déterminer le statut argumentatif de chaque fait de la liste complète. Ensuite des règles spécialisées sont appliquées afin de décrire comment les faits seront insinués ou justifiés, et comment des «normes coutumières» seront utilisées pour renforcer l'argumentation. Après application de ces règles, on obtient un premier ensemble de faits qui sont candidats pour être explicitement évoqués dans la description argumentée. La dernière étape consiste à 
appliquer des règles rédactionnelles, et des règles de raffinement lexical et syntaxique afin de produire la description argumentée sous la forme la plus convaincante possible. Nous examinons dans ce qui suit chaque catégorie des règles constituant notre système

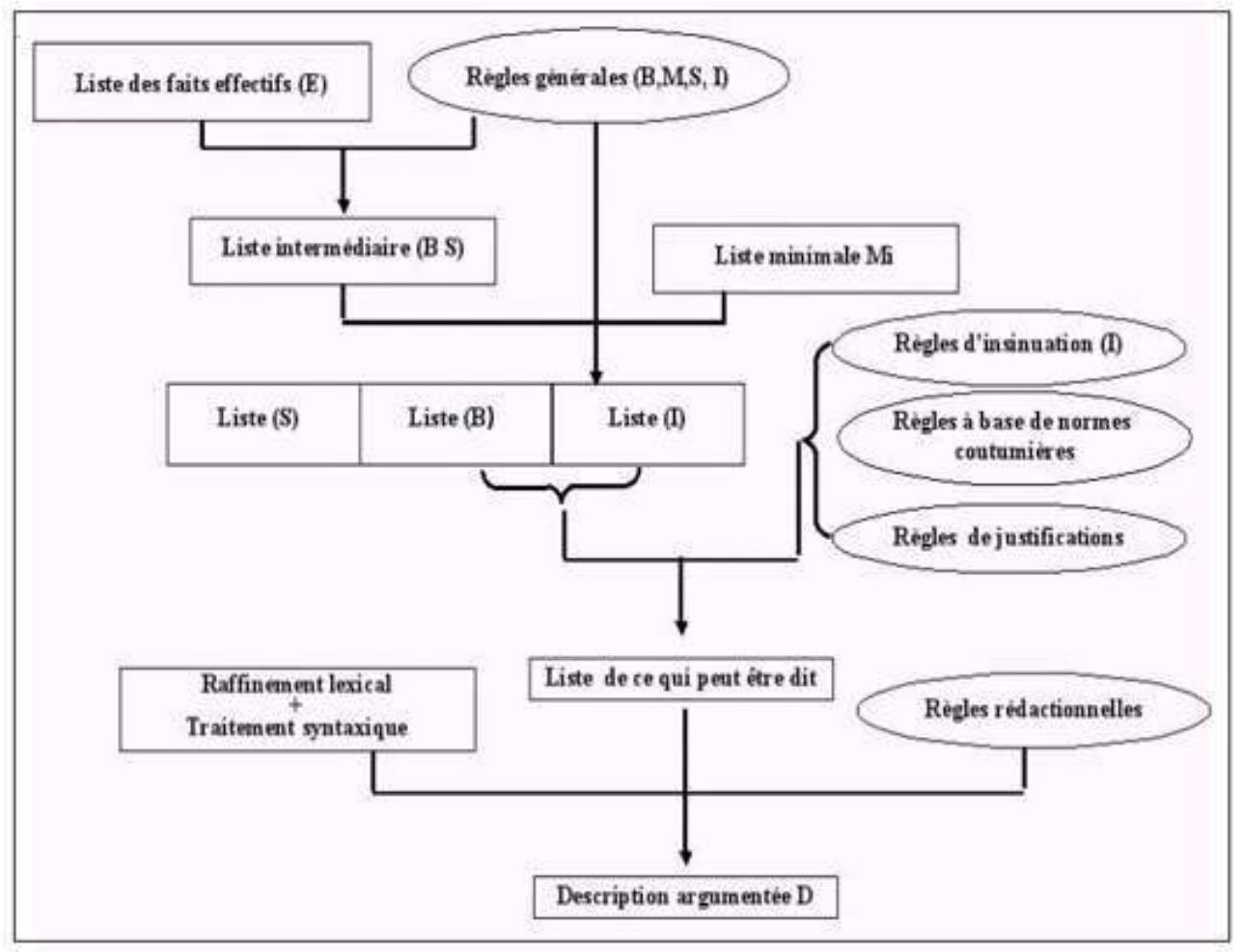

Figure 1. Architecture générale du système d'argumentation

$E=$ fait effectif, i.e. supposé être produit dans la situation décrite ; $B=$ =élément favorable à l'auteur (a priori, Bon à dire); $M$ =élément défavorable à l'auteur (Mauvais à dire) ; $S$ = élément défavorable qu'il vaut mieux taire (Silence); $\mathrm{Mi}$ = fait dont nous jugeons la présence indispensable dans toute description de l'accident (liste Minimale)

\subsubsection{Règles générales déterminant ce qui est bon ou mauvais à dire $(B, M, S, I)$}

Cette catégorie de règles générales permet dans un premier temps de placer chaque action ou état figurant dans la liste des faits effectifs dans son contexte afin de déterminer s'il est bon ou mauvais de l'exprimer dans la description argumentée. Les principes de la conduite du code de la route mais aussi les normes de bon sens représentent la source des connaissances nécessaires pour effectuer ce raisonnement: si une action ou un état est conforme aux règles du code de la route ou correspond du moins à ce qu'on s'attend généralement à voir une personne faire dans un contexte donné, on peut le considérer comme bon sinon, on le considère comme mauvais.

\section{Exemples}

- Il est bon de garder une distance de sécurité par rapport au véhicule précédent.

- Il est bon d'éviter les obstacles.

Ici, les faits cités sont bons indépendamment de toute situation particulière. Mais on peut aussi avoir des règles contextuelles qui permettent de déduire si un fait est bon ou mauvais. Par exemple :

- Reculer sur sa file n'est pas bon en général, mais si on le fait pour faciliter la manœuvre d'un poids lourd, c'est une bonne chose. 
Cet exemple est un cas particulier d'une règle abstraite ou d'un schéma de règles qu'on peut exprimer ainsi: Si les circonstances font qu'une personne doit effectuer une action A, alors le fait qu'elle l'effectue est jugé bon.

Dans un deuxième temps, les règles générales permettent de répartir les faits qui ont été jugés mauvais à dire en deux classes : ceux qu'il vaut mieux passer sous silence (S) et ceux qu'on ne peut pas passer sous silence mais qu'il faudra essayer d'insinuer sans les évoquer explicitement. Le critère de répartition est simple: si un fait jugé mauvais à dire n'appartient pas à la liste minimale, il suffira de ne rien dire à son propos dans la description argumentée, sinon les règles suggèrent qu'une tentative d'insinuation doit être faite.

\subsubsection{Règles d'insinuation}

27 Nous sommes parfois dans l'obligation de dire certains faits qui ne nous arrangent pas et qui risquent d'affecter gravement notre argumentation. C'est le cas, comme nous l'avons déjà expliqué, des faits mauvais à dire mais appartenant à la liste minimale. Les règles d'insinuation nous permettent alors de faire indirectement comprendre que ces faits ont eu lieu, et de les présenter d'une manière souple et atténuée, sans choquer le lecteur par leur gravité. Nous exploitons entre autres les sous-entendus pour faire passer d'autres informations qui nous arrangent.

28 Une des stratégies consiste à exploiter certaines modalités (intention, croyance, ...) qui laissent entendre le respect de certaines normes sans affirmer explicitement que tel était bien le cas. L'utilisation des croyances et d'intentions permet de semer un doute sur la réalisation ou pas des actes matériels exprimés en fonction de ces dernières. Il est clair qu'une intention d'entreprendre une action n'implique pas forcément son accomplissement, c'est ce qui nous laisse une marge de manœuvre pour jouer en faveur de l'auteur. Celui-ci ne sera jugé en fin de compte que sur les actions qu'il a faites et celles qu'il n'a pas faites bien qu'il eût dû les faire.

\section{Exemples}

- Écrire : «En sortant de mon domicile,...» permet d'inférer que l'auteur n'était pas prioritaire par rapport à un autre véhicule se trouvant sur la voie de circulation. Et si un accident s'est produit, cette inférence permet de penser qu'il n'a pas respecté cette priorité.

- Écrire: «...Voulant tourner à gauche...» sous-entend que l'auteur a mis son clignotant à gauche, sans affirmer qu'il l'a réellement fait, et le déroulement de l'accident permet justement d'inférer qu'il ne l'a pas fait.

\subsubsection{Règles à base de normes coutumières}

Pour argumenter, nous faisons souvent appel à des «normes coutumières» (appelées également règles informelles). Les normes coutumières sont des règles de comportement qui ne figurent dans aucun code, mais qui sont très largement reconnues comme légitimes. Cette légitimité est d'ailleurs souvent implicitement invoquée dans notre corpus comme une excuse pour justifier la violation d'une vraie norme. Des études sociopsychologiques ont été effectuées sur les comportements des conducteurs de voitures dans des intersections afin de déterminer à quel point l'utilisation de ces règles informelles est influencé par des paramètres divers comme l'âge, le sexe, la profession, la position du sujet dans l'intersection, ... (Gunilla, 2005). 


\section{Exemple de norme coutumière}

- Si A est prioritaire sur B dans une intersection mais qu'il n'exerce pas sa priorité $\mathrm{c}$-à-d s'il tarde à s'engager, alors $\mathrm{B}$ considère que $\mathrm{A}$ n'est plus prioritaire et qu'il devient légitime pour lui de s'engager.

La stratégie argumentative de certains auteurs consiste à justifier la violation d'une norme «forte», (e.g, s'arrêter au feu rouge, ne pas démarrer si on a un obstacle devant soi, ...), par le fait que l'adversaire n'a pas respecté une norme «faible» (e.g. démarrer quand le feu passe au vert).

Une illustration de cette stratégie vis-à-vis de la norme coutumière énoncée ci-dessus est fournie par le texte suivant (texte D45 de notre corpus) :

- La sortie de la rue Nom_De_Voie était embouteillée : le véhicule sur ma droite étant arrêté, j'ai cru qu'il me laissait passer. J'ai mis mon clignotant et je me suis engagée, mais le conducteur de la voiture A s'est finalement décidé à avancer, d'où le choc.

L'auteur reconnaît que son adversaire avait la priorité, mais comme il ne l'a pas exercée immédiatement, il s'est considéré en droit de la lui refuser: le non-respect par l'adversaire de la norme faible «quand on a la priorité, on doit l'exercer immédiatement» sert d'argument à l'auteur pour violer la norme forte «on doit respecter la priorité à droite».

Comme cette stratégie argumentative apparaît assez convaincante, au moins pour un lecteur non prévenu, certains auteurs vont jusqu'à sous-entendre l'existence d'une norme coutumière - qu'ils ont en fait créée pour les besoins de la cause - afin de justifier un comportement autrement difficilement excusable.

Nous trouvons par exemple dans notre corpus (texte B46) :

- Je venais du Boulevard Nom_De_Voie1, le feu était au vert et deux flèches au sol m'indiquaient que les voies sur lesquelles je me trouvais me permettaient de tourner à gauche. Pensant avoir la priorité, je me suis engagée dans la direction de la place Nom_De_Voie2 lorsque j'ai heurté la Nom_De_Véhicule de Mr Nom_De_Personne.

L'auteur invoque une norme selon laquelle la présence de flèches au sol confère une priorité ; il reconnaît que cette norme n'existe pas vraiment, mais c'est la meilleure excuse qu'il ait trouvée pour expliquer son comportement.

\subsubsection{Règles de justification}

Il y a parfois des faits qui ne nous arrangent pas, qui figurent dans la liste des faits qui doivent être évoqués, et pour lesquels nous ne disposons pas de stratégie d'insinuation. Dans ce cas, nous présentons explicitement ces faits et nous avons recours à des justifications pour tenter de convaincre le lecteur qu'ils sont quand même légitimes. Parmi les méthodes avec lesquelles on peut se justifier :

\section{Absence d'alternatives :}

Il s'agit de présenter les choses comme s'il n'y avait pas d'autre choix à faire, ou que l'auteur avait opté pour la meilleure solution possible pour minimiser des dégâts qui n'auraient pu être que plus graves s'il avait choisi une alternative. Par exemple, dans «J'ai dû braquer pour éviter un véhicule». L'emploi du verbe devoir a pour but de faire comprendre au lecteur que le braquage, qui n'est pas forcément en faveur de l'auteur, était la seule manœuvre qu'il pouvait exécuter afin d'éviter un choc plus grave que celui décrit dans 
l'accident. Cet argument peut masquer le fait que, s'il s'est retrouvé devant cette alternative, c'est probablement parce que l'auteur avait commis une erreur auparavant (p.ex. s'engager sans regarder).

\section{Utilisation d'une relation cause/conséquence :}

La relation causale permet de justifier un accident par une seule de ses causes, celle qui l'exonère au maximum de ses responsabilités. Par exemple, dans un passage du texte B69: «Mon arrêt au stop n'était pas tout à fait rigoureux (chaussée mouillée).", l'auteur laisse entendre qu'il n'est pas responsable de la pluie, qui est certes un facteur d'allongement des distances de freinage, donc une cause de son arrêt " pas tout à fait rigoureux » ... pour ne pas dire que l'état de la chaussée était parfaitement connu de lui, et qu'il n'en a pas tenu compte.

\section{Argumentation basée sur l'information disponible:}

De nombreux auteurs d'accidents essaient de se justifier en expliquant au lecteur qu'ils ne disposaient pas de tous les paramètres de la situation au moment des choix décisifs. Ils ne peuvent cacher que ces choix se sont avérés malheureux et ils en explicitent les conséquences, mais ils montrent qu'au moment où ils les ont faits, l'information en leur possession n'était pas celle qu'ils ont acquise par la suite. La technique utilisée consiste à recourir à certaines modalités :

\section{- La croyance:}

Les auteurs peuvent justifier leurs choix malheureux en arguant qu'il était basé sur une information fausse, mais qu'ils croyaient alors exacte. Par exemple (texte D24) dans : « La voiture B, croyant que A pouvait passer, s'est avancée», l'auteur (notons qu'il s'assimile à sa voiture !) dit explicitement qu'il s'est avancé (dans le contexte, ce n'est pas en sa faveur, car le passage est très étroit), mais il tente de le justifier par sa croyance de pouvoir le faire sans gêner l'adversaire, ce qui était bien entendu faux.

\section{- L'intention:}

L'intention est une attitude d'un agent relativement à une action quand il décide, suite à une évaluation rationnelle, d'entreprendre cette action. Les intentions sont largement utilisées pour justifier des choix qui se sont révélés inappropriés. L'idée est de montrer la rationalité et/ou la bonté des intentions de l'auteur, et que les conséquences indésirables n'étaient pas du tout dans le plan ou les objectifs de l'auteur.

Par exemple (texte D3) dans : «Je virais à droite pour aller stationner lorsque je suis rentré dans un véhicule ", l'auteur motive son virage à droite qui a conduit au choc par son intention tout à fait rationnelle d'aller stationner son véhicule ; et (texte B72) dans « Voulant éviter un chien qui traversait, j'ai heurté le mur ! ", l'auteur avance son intention louable d'éviter de heurter le chien comme justifiant le fait qu'il a heurté le mur. 


\subsubsection{Règles rédactionnelles}

\subsubsection{Filtrage}

l'implication du protagoniste qu'on défend dans la cause de l'accident. Nous parviendrons à cet objectif de deux façons non exclusives : minimiser la responsabilité de ce dernier et maximiser la responsabilité de son adversaire. L'analyse des textes de notre corpus nous a permis de constater que certains auteurs mettent trop d'insistance à accuser leurs adversaires ou accentuent exagérément certains éléments, ce qui finit par susciter un doute sur la véracité de leur relation des événements. Mettre trop d'informations, même si elles sont vraies, risque de nuire au but de l'argumentation. Une tâche importante est ainsi la sélection des éléments qui vont figurer dans la description argumentée parmi ceux qui peuvent être dits. Trouver ce compromis est loin d'être une tâche facile vu que le fait de juger si un fait vient ou non surcharger l'argumentation nécessite l'interaction d'informations diverses.

\section{Exemple}

Supposons que la liste des propositions contienne le fait que «je» roulais derrière un conducteur qui conduisait rapidement, et qui a freiné brutalement. Ces deux adverbes sont en défaveur de l'adversaire, et entrent donc dans la catégorie des «choses bonnes à dire ".

Cependant, une analyse plus profonde permet d'écarter la qualification de la vitesse et ne garder que celle du freinage. En effet, puisque l'auteur était derrière son adversaire, un lecteur ordinaire conclura que l'auteur roulait à une vitesse proche de celle de ce dernier, et laisser entendre qu'elle était excessive ne nous arrange pas.

\subsubsection{Ordonnancement des faits}

L'ordre dans lequel les éléments de la description sont disposés est un facteur majeur pour la construction d'une bonne argumentation. Doit-on privilégier l'aspect logique ou chronologique? Nous avons constaté, en analysant les textes, que les auteurs ont recours à différentes stratégies d'ordonnancement, selon qu'ils se considèrent ou non fautifs, dans le but de présenter les choses sans choquer le lecteur par la gravité des erreurs qu'ils ont commises, et surtout pour ne pas mettre ces erreurs en relief. Souvent la simple juxtaposition dans l'ordre chronologique suffit à créer, entre deux propositions, plusieurs types de rapports : addition, consécution, opposition et parfois cause/conséquence.

Une stratégie qui nous paraît utile, dans le cas où l'auteur est fautif, consiste à exprimer en premier lieu l'intention des actions entreprises afin de susciter la confiance du lecteur et de lui faire partager son point de vue. Ensuite, on enchaîne par la description de la faute commise, et enfin on termine par la description du choc (en le minimisant ou en le maximisant selon qui en est présenté comme responsable). D'autres facteurs participent à l'ordre «optimal » selon les règles utilisées au cours des traitements qui imposent elles aussi des enchaînements particuliers : prémisses - conclusions par exemple dans le cas des règles de justification. 


\subsubsection{Raffinement lexical et traitement syntaxique} sémantique des verbes, des adjectifs ou des adverbes qui expriment les mêmes actions. Les éléments de chaque classe sont ordonnés selon une échelle de force de leurs expressions, que l'on utilisera du plus fort au plus faible ou inversement en fonction du protagoniste qu'on défend:

- Action_choc : est une classe qui regroupe toutes les actions décrivant un choc. Exemple : heurter, cogner, percuter, toucher, ..., etc. Chacun de ces verbes exprime une action de choc, mais certains verbes connotent cette action avec plus de violence que d'autres; ces derniers sont des candidats préférés quand on veut exprimer une action de choc effectuée par l'adversaire. Par exemple on préfère dans de tels cas un verbe comme « percuter » qu'un verbe comme « toucher».

- Vitesse1 : est une classe qui regroupe les adjectifs et les adverbes qualifiant une vitesse modérée. Par exemple : modérée, normale, réglementaire, lentement, etc.

- Vitesse2 : est une classe qui regroupe les adjectifs et les adverbes qualifiant une grande vitesse. Par exemple : vite, trop_vite, rapidement, vive_allure, etc.

- Qualif3 : est une classe qui regroupe les adverbes qualifiant un choc violent. Par exemple : violemment, brusquement, brutalement, fortement, gravement, etc. Ainsi, au lieu de dire « heurter » tout court, on lui adjoindra un adverbe du type Qualif3 et on dira : «heurter violemment», ce qui permet de sous entendre entre autres : « il roulait vite ", " la voiture est très abîmée ", " il est responsable». 
- Qualif4 : est une classe qui regroupe les expressions adverbiales qualifiant l'action de freinage. Par exemple : sec, net, subitement, soudainement, sans_raison, tardivement, sans_prévenir.

- Qualif5 : est une classe qui regroupe les adverbes qui qualifient un choc léger. Par exemple : légèrement, à_peine,...

- Qualif6 : est une classe qui regroupe les adjectifs qui qualifient l'état d'une partie de véhicule sérieusement endommagée par un choc. Par exemple : enfoncée, très enfoncée, ébréchée, détériorée, endommagée, très endommagée, cassée, abîmée, très abîmée.

51 Outre ces techniques lexicales, il existe aussi des possibilités offertes par la syntaxe pou l'argumentation, notamment celle qui consiste à utiliser la voix passive à la place de la voix active pour parler d'une "mauvaise » action effectuée par l'auteur. La voix passive sous-entend une part très faible d'initiative de l'auteur dans l'exécution de l'action décrite, ce qui minimise sa responsabilité. Par exemple, au lieu de dire "J'ai dérapé et ce faisant j'ai heurté l'autre véhicule ", on préfère dire : "J'ai été déporté, ce qui m'a fait heurter l'autre véhicule».

\section{Un langage de représentation de connaissance}

\subsection{Le langage de représentation}

Nous avons opté pour un langage de programmation logique du premier ordre réifié Selon cette technique, un prédicat ternaire $P(x, y, z)$ qui exprime le fait que la propriété $P$ s'applique à trois arguments $x, y$ et $z$ sera écrit après réification : vrai $(P, x, y, z)$. Le nom de prédicat $P$ devient alors un argument du nouveau prédicat vrai.

Cette technique permet de faire des abstractions sur les noms des prédicats en les désignant par des variables susceptibles d'être quantifiées dans les règles d'inférence. En revanche, un de ses inconvénients est qu'elle force une arité fixe du prédicat vrai. Afin de résoudre ce problème, nous introduisons une fonction binaire spécifique combine, qui prend deux arguments et construit un argument complexe qui consiste en leur combinaison. La réification d'une propriété quadruplet $Q(x, y, z, t)$ donnera (par exemple)

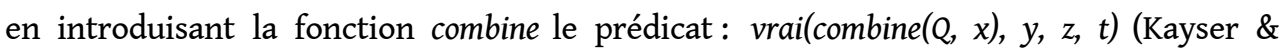
Nouioua, 2004).

Dans notre système, le prédicat vrai s'applique à un quadruplet, et il est de la forme : vrai $(X, P, A, T)$ où :

- $X$ est une étiquette qui permet d'exprimer le statut du prédicat vrai pour chaque niveau de traitement; entre autres, elle permet d'affecter le statut argumentatif des faits. $X$ peut prendre plusieurs valeurs, chacune donne une signification spéciale pour ce prédicat.

1. E, la propriété $P$ est effectivement vraie.

2. $B$, il est bon, si on argumente en faveur de l'agent $A$, de dire que la propriété $P$ est vraie.

Corela, 6-1 | 2008 

l'interprétation par intervalles ou par instants: la seule chose qui importe est de
décomposer la scène de l'accident en une succession d'éléments ordonnés temporellement. Chaque élément est dénoté par un nombre entier représentant son ordre, et c'est lui qui est employé comme argument. La signification de l'élément dépend de la nature de la propriété. Si c'est une propriété persistante, le paramètre temporel dénote un intervalle de temps pendant lequel cette propriété demeure vraie (sémantique basée sur les intervalles). Si la propriété n'est pas persistante (elle correspond à une action ou à un événement ponctuel), alors l'argument temporel dénote le point de départ de l'intervalle sur lequel la propriété se produit et fait changer la valeur de vérité d'au moins une propriété persistante.

\subsection{Les modalités}

68 Comme nous venons de le signaler, les futurs «latents» sont pris en compte par l'intermédiaire de modalités. Dans notre système, nous avons besoin d'exprimer plusieurs modalités :

- La modalité devoir exprime une sorte de nécessité et permet de rendre compte des devoirs des agents.

- La modalité en_mesure exprime une sorte de possibilité, elle permet de traiter les capacités des agents.

- La modalité intention exprime les intentions de l'agent. Elle permet aussi d'exprimer des états du monde que l'agent a souhaité atteindre sans succès.

Corela, 6-1 | 2008 
- La modalité croire exprime les croyances de l'agent sur le monde à un moment donné.

la capacité de pouvoir traiter les exceptions, ce qui implique de remettre en cause certaines conclusions à la lumière de nouvelles informations (McCarthy, 1980). La logique ordinaire n'est donc pas adéquate pour rendre compte de ce type de raisonnement, et en particulier pour le raisonnement argumentatif.

75 Parmi plusieurs formalismes de représentation du raisonnement de sens commun, nous avons choisi le formalisme de la «logique du raisonnement par défaut » (Reiter, 1980), pour la représentation de nos règles d'inférences. La sémantique de point fixe qui caractérise cette logique est adéquate au processus de compréhension de la langue naturelle. En effet, une «théorie » dans cette logique peut avoir de multiples extensions, qui peuvent correspondre à différentes argumentations possibles partant d'une même description effective.

76 Une théorie au sens de cette logique est formée de deux composantes : un ensemble de formules de logique classique, dans lequel on peut donc introduire des implications matérielles, pour représenter les inférences qu'on ne souhaite jamais remettre en cause, et un ensemble de défauts, pour représenter les inférences dont les résultats peuvent être annulés.

\subsection{Implémentation via Answer Set Programming}

77 Afin de mettre en œuvre notre langage de représentation, nous avons opté pour le paradigme de la « programmation par ensemble de réponses » (Answer Set Programming, 
Gelfond \& Lifschitz, 1991). ASP utilise le concept de négation par échec qui permet de résoudre des problèmes avec des connaissances par défaut et ainsi de réaliser un raisonnement non-monotone. Il permet aussi d'exprimer des exceptions, des restrictions et de représenter une connaissance incomplète.

Plusieurs outils ont été développés permettant d'écrire des programmes dans le paradigme ASP. Nous avons utilisé pour notre système le langage Smodels ${ }^{2}$ et nous avons traduit nos règles d'inférences en termes de règles de ce langage. Smodels permet de calculer les "modèles stables». Un modèle stable contient l'ensemble des littéraux appartenant à une extension de la théorie des défauts correspondante.

\section{Exemple :}

Soit la règle écrite en logique des défauts, qui exprime : «par défaut, passer sous silence le fait que la personne a heurté un obstacle sauf si cette information appartient à la liste minimale » :

vrai( $M$, combine(heurter, Obstacle), Personne, $T$ ) : vrai (S, combine(heurter, Obstacle), Personne, $T$ ) [-vrai(Mi, combine(heurter, Obstacle), Personne, T)].

Lire: s'il est mauvais de dire (statut argumentatif $M$ ) que la personne que l'on défend a heurté un obstacle au temps $T$, alors affecter le statut argumentatif $S$ (silence) à ce fait, sauf preuve $d u$ contraire, et sauf si ce fait appartient à la liste minimale (statut argumentatif Mi).

Traduction de cette règle en Smodels :

vrai (s, combine(heurter, 0$)$, personne, $T)$ :- instant $(T)$, obstacle $(0)$, vrai ( $m$, combine(heurter, $O)$, personne, T), not vrai(mi, combine(heurter, 0$)$, personne, $T)$, not -vrai (s, combine(heurter, 0$)$, personne, T).

Lire: une façon de déduire qu'il vaut mieux passer sous silence le fait que la personne a heurté 0 en $T$ consiste à prouver que $T$ est un instant, que $O$ est un obstacle, que ce fait est mauvais à dire, que l'on n'a pas la preuve qu'il appartient à la liste minimale [not vrai $(m i, . .)$.$] et que l'on n'a pas la$ preuve qu'il ne doit pas être passé sous silence [not-vrai $(s, \ldots)]$.

\section{Conclusion et perspectives}

Comme il a été brièvement rappelé en introduction, travailler sur l'argumentation n'est pas très original! De nombreux chercheurs l'ont fait depuis des siècles, dans des cadres de pensée très variés. Les informaticiens ont jusqu'ici principalement cherché à construire des systèmes incarnant les principes logiques qui sous-tendent le raisonnement argumentatif et / ou des systèmes de dialogue orientés pour la résolution de tâches précises (p.ex. une réservation touristique). Mais ils n'ont pas, à notre connaissance, abordé le problème qui nous intéresse ici : présenter un événement d'une façon qui serve au mieux les intérêts d'un de ses protagonistes.

87 Le travail que nous avons entrepris présente donc l'originalité, vis-à-vis des informaticiens, d'être moins concerné par les préoccupations logiciennes et beaucoup plus par des phénomènes liés à la langue; et vis-à-vis des Sciences du langage, de ne pas se limiter à une analyse des procédés argumentatifs, mais d'envisager la construction effective d'un système fabriquant une description argumentée.

Ce que nous en avons présenté dans cet article ne correspond cependant qu'à une première phase: choix généraux de l'architecture logicielle, analyse préalable de quelques stratégies identifiables dans le corpus recueilli. La phase suivante consiste à 
implémenter les différentes règles identifiées dans notre architecture (cf. Figure 1). Cette phase est actuellement en cours, et donne déjà quelques résultats encourageants.

Plus délicate sera la phase de validation : après que nous aurons testé ces règles sur un nombre significatif d'exemples, et que nous les aurons affinées pour qu'elles fournissent des descriptions argumentées qui nous paraîtront satisfaisantes, comment pourrons-nous donner un contenu moins subjectif à cette satisfaction? La solution envisagée consiste à bâtir à partir des mêmes faits plusieurs descriptions argumentées, et à les soumettre à des évaluateurs humains : nous verrons alors comment leur jugement place la description fournie par notre système (transformée, probablement manuellement, en un texte en français convenable) par rapport à celles construites par d'autres rédacteurs, y compris le rédacteur du texte réel dont nous sommes partis.

Dans une phase ultérieure, et en supposant que les résultats de la validation soient favorables, nous pourrons envisager d'extraire de nos règles celles qui ne dépendent pas du domaine traité, en vue de les incorporer dans un système générique d'aide à la rédaction de textes argumentés.

Remerciements : Nous remercions vivement Françoise Gayral et Thierry Poibeau et les relecteurs de la revue Corela pour leurs commentaires sur une première version de cet article. Ce travail a été entrepris dans le cadre, et avec le soutien financier, du projet MICRAC de l'Agence Nationale de la Recherche.

\section{BIBLIOGRAPHIE}

Anscombre J.C. \& Ducrot O., 1983 : L'argumentation dans la langue. Bruxelles : Editions Mardaga.

Amgoud L., Bonnefon J.F. \& Prade H., 2005 : An argumentation-based approach to multiple criteria decision. In 8th European Conference on Symbolic and Quantitative Approaches to Reasoning with Uncertainty, Barcelone, juin 2005. Actes LNCS, pp. 269-280.

Baker M. J., 1994 : Argumentation, Explication et Négociation : analyse d'un corpus de dialogues en langue naturelle écrite dans le domaine de la médecine. Dans Modélisation d'explications sur un corpus de dialogues : Actes de l'atelier de recherche, pp. 1-26. Rapport de Télécom Paris, $\mathrm{N}^{\circ}$ 94-S-003.

Das S.K., Fox J. \& Krause P., 1996 : A unified framework for hypothetical and practical reasoning (1) : Theoretical foundations. In International Conference on Formal and Applied Practical Reasoning, Londres, Proceedings pp. 58-72. Springer-Verlag.

Ducrot O., 1980 : Les Échelles Argumentatives. Paris : Minuit.

Dung P.M., 1995. On the acceptability of arguments and its fundamental role in nonmonotonic reasoning, logic programming and n-person games. Artificial Intelligence Journal, 77(2), pp. 321357.

Garcia A., Gollapally D., Tarau P. \& Simari G., 2000 : Deliberative stock market agents using JINNI (Java INference engine and Networked Interactor) and defeasible logic programming. In 
European Conference on Artificial Intelligence, Workshop on Engineering Societies in the Agents' World, Berlin, août 2000. Springer Verlag.

Gelfond M. \& Lifschitz V, 1991 : Classical negation in logic programs and disjunctive databases. New Generation Computing, 9(3-4), pp. 363-385.

Grize J.B., 1990 : Logique et langage. Ophrys, Paris.

Gunilla B, 2005 : Driver Interaction: Informal Rules, Irritation and Aggressive Behaviour. Digital Comprehensive Summaries of Uppsala Dissertations from the Faculty of Social Sciences 8.

Kayser D. \& Nouioua F., 2004 : Representing Knowledge About Norms. In 16th European Conference on Artificial Intelligence, Valence (Espagne), août 2004, pp. 363-367.

Lempereur. A., 1991 : L'Argumentation. Liège, Éditions Mardaga.

McCarthy J., 1980 : Circumscription : A form of non-monotonic reasoning . Artificial Intelligence Journal, 13(1-2), pp. 27-39.

Mel'cuk I.A., Clas A. \& Polguère A., 1995 : Introduction à la lexicologie explicative et combinatoire. Éditions Duculot. Coll.Champs linguistiques.

Perelman C. \& Olbrechts-Tyteca L., 1985 : Traité de l'argumentation. Paris : Presses Universitaires de France.

Plantin C., 1990: Essais sur l'argumentation. Introduction à l'étude linguistique de la parole argumentative. Paris : Éditions Kimé.

Prakken H., 1997 : Logical tools for modeling legal argument : A study of defeasible reasoning in law. In 4th Workshop on formal Models of Agents, Actes pp. 201-214.

Prakken H \& Sartor.G., 1996 : A dialectical model of assessing convicting arguments in legal reasoning. Artificial Intelligence and Law, 4, pp. 331-368.

Reiter R., 1980 : A Logic for Default Reasoning. Artificial Intelligence, 13 (1-2), pp. 81-132.

Sycara K., 1989 : Argumentation : Planning other agents' plans. In 11th International Joint Conference on Artificial Intelligence, Detroit, USA, pp. 517-523.

Toulmin S.E., 1985 : The Uses of Argument. Cambridge University Press, Cambridge, UK. Tal, 1994 : Numéro spécial : Approches sémantiques. Traitement automatique des langues 35(1).

\section{NOTES}

1. Ce corpus, obtenu grâce à la compagnie d'assurance MAIF que nous remercions ici, contient 160 rapports que nous avons anonymisés; il est disponible sur la page http://www-lipn.univparis13/ kayser/corpus160.pdf

2. En fait, un premier logiciel appelé Lparse permet entre autres d'instancier les variables afin de produire des règles propositionnelles. Smodels opère ensuite sur le résultat du traitement des règles par ce logiciel. Lparse et Smodels sont disponibles sur le web à l'adresse: http:// www.tcs.hut.fi/Software/smodels/ 


\section{RÉSUMÉS}

L'argumentation se situe à la croisée de disciplines diverses : pragmatique de l'énonciation, rhétorique, logique formelle, compréhension des textes. Elle constitue un champ d'études à la fois descriptif et critique qui s'intéresse à la mise en forme des arguments (oralement ou par écrit) en vue, notamment, de la persuasion d'un auditoire. Nous nous intéressons ici à l'argumentation exprimée dans un cadre naturel (textes), avec pour objectif la production de « descriptions argumentées ", c'est-à-dire de descriptions qui, sans travestir la réalité, déclenchent chez le lecteur des inférences le conduisant finalement à conclure ce qui «arrange» l'auteur. L'enjeu sera donc d'étudier les stratégies permettant de générer de telles descriptions. Dans cet article, nous mettons l'accent sur l'analyse de descriptions existantes (les rapports figurant dans les constats d'accident) afin de mettre en évidence des stratégies qui nous serviront, dans un deuxième temps, pour la génération de descriptions argumentées.

Argumentation is situated at the crossroads of various disciplines; it concerns formal logic, as well as the production of texts appropriate to a given communicative situation, and even the interpretation of texts. The argumentation is a field of study, both descriptive and critical, concerned with the layout of the arguments (orally or in writing), notably in view of persuading an audience. In our work, we are interested in the expression of arguments in their natural framework (texts), with the aim of producing "biased descriptions", i.e. descriptions which, while remaining truthful, trigger in the reader's mind inferences ultimately leading him / her to conclude what is in "favour" of the author. The challenge will be to find strategies that generate such descriptions. In this article, we focus on the analysis of existing descriptions (the textual part of car crash reports) to highlight strategies used by human writers. We will take advantage of these strategies, in a second stage, to generate automatically "biased descriptions".

\section{INDEX}

Mots-clés : argumentation, intelligence artificielle, raisonnement, langage naturel, stratégies argumentatives

Keywords : argumentation, Artificial Intelligence, reasoning, natural language, argumentative strategies

\section{AUTEURS}

\section{SARA BOUTOUHAMI}

Université Paris-Nord, 93430 Villetaneuse, LIPN UMR 7030 du CNRS. Institut Galilée

DANIEL KAYSER

Université Paris-Nord, 93430 Villetaneuse, LIPN UMR 7030 du CNRS. Institut Galilée 